\title{
Strategic Design and UX Design Approaches in the Development of Fashion Design Systems-Products
}

\author{
Paula Cristina Visoná ${ }^{*}$ and Hallana Gomes De Souza ${ }^{2}$ \\ ${ }^{1}$ Professor, Design and Fashion course, University of Rio dos Sinos Valley (Unisinos), Brazil \\ ${ }^{2}$ Graduate Student, University of Rio dos Sinos Valley (Unisinos), Brazil
}

*Corresponding author: Paula Cristina Visoná, Professor, Design and Fashion course, University of Rio dos Sinos Valley (Unisinos), Brazil.

\begin{abstract}
This paper aims the study and systematization of the projectual approaches of the Strategic Design and UX Design through its correlation with Fashion Design, in order to propose a systemically model that helps the creation of a Fashion Brand and the nutrition of its development cycle of product, services and communication.
\end{abstract}

Keywords: System-product; Strategic design; UX sesign

\section{Introduction}

Our study characterizes and introduces the Strategic Design and UX Design tools and establishes them as resources usable in the fashion creative chain, especially on their design thinking, the pursuit of innovation and the construction of a self-nourishing brand-building cycle.

To connect each of these points and achieve these goals, it is necessary to highlight the relationship between these different fields of design, as well as the importance of the designer and user in this process

\section{Strategic Design and UX Design}

It is through reflection on Transdisciplinarity and its role that we can understand the strategic function of design in a business, and this has a lot to do with increasing the value of brand image and its founding pillars. The key to this consideration begins with understanding what design is and how it branches into other areas. A first understanding can be taken from the thermogravimetric analysis, that derived from the Latin designare (design) which, according Krippendorff $\mathrm{K}$ [1] is composed of (preposition linking two nouns) and signum (sign, signal, image, etc. ), which may be equivalent to drawing and projecting. However, design is much deeper than that, once allows the creation of something and its significance, creating meaning from a semiotic relationship.
Also in this sense, Moraes [2] states that the design has become a major player when it comes to contemporary projectual disciplines and that this is due to its transversal and multidisciplinary nature, which makes it able to deliver quick answers through "products , images and new forms of interaction" [2].

We also highlight the thought of Flusser that design is the place where "art and technique (and, consequently, value and scientific thoughts) go together, with equivalent weights, making a new form of culture possible ", when related to other design disciplines such as architecture, fashion and engineering, it is understood as a design culture [3] and presents itself as a set that articulates knowledge of different competences, which develops from practical of reflection in action.

Thus, there is the project culture, a field that deals with and highlights the importance of building an inter and transdisciplinary approach to knowledge, constituting new ways of thinking and design. At the same time, the project culture develops also from the connection between the areas that constitute a company design, administration, marketing, production, etc. - providing the integration of the project activity and aligning the same visions to a same goal, so that one area can contribute to another as an organism working strategically to develop results and innovation for the entire system-product-service of the organization. 
Since design is a multidisciplinary and cross-sectional discipline, which has specialized and joined some specific areas, such as Strategic Design and UX Design, we will deepen the study of each of these specialties, pointing out why their choice and how they influence the development of a fashion-driven design culture. Thus, by Strategic Design we have: [...] the project activity co-opted in the formulation and development of an organization's strategies; Its goal is to shape the strategy that is primarily a product system, that is, an organic and coherent set of the various means (product, service, communication) with which the company builds its own identity, positions itself in the market, defines the meaning of its mission in society [4].

Another way to understand what strategic design is by analyzing the term strategy. Minstzberg et al. [5], explain that the word can be interpreted as plane and pattern, positions and perspective and trick. The first two definitions being the most widespread, understanding them as the principle that indicates paths and routes designed to achieve some goal, which arises from an analysis of some current or past consistent behavior. As for position and perspective, strategy can be understood as something that analyzes external factors - cultural, behavioral and market patterns - and absorbs from its directions that will define the characteristics of the organization. Finally, strategy as a trick can be understood as using trickery and reaction maneuvers for potential threats.

As part of its system, Strategic Design demands a practice known as pre-project or meta-project, which, according to Moraes [2] configures into something: [...] that goes beyond the project, because it transcends the project act. This is a preliminary critical and reflective thinking about the project itself from a scenario assumption on which stand the productive factors, technological, marketing, materials, environmental, socio-cultural and aestheticformal, with the analyzes based and reflections previously performed prior to the project phase, through prior and strategic data collection.

The author also points out that the metaproject has a comprehensive character that aims to explore the potential of design through the non-production of a single model and pre-established techniques, being fluid and dynamic and acting as a transformative agent of the human, social and technological context [6]. Another important point to note is that the metaproject considers different processes, especially regarding research in terms of behavior, changing scenarios, culture, technologies, expertise, competition, among others. That is, "the metaproject considers the product far beyond the product design, but also as a linguistic character and its emotional implications during use [2].

We can define the metaprojetual phase as a problematic stage (or briefing), reflection, analysis and interpretation, experimentation and definition on the approaches to be adopted to solve this problem, which requires a qualitative approach which helps to identify the main points of briefing the be explored. The selection of signs and references that contribute to the search for possible answers to be developed in the project, the stimulation of creativity and the interpretation of possibilities are perspectives contemplated in the metaprojectual phase.

In this process of exploring possibilities, the meta-project also suggests the construction of scenarios that help to foresee the future. This tool serves as a creative input to design not to shape the future but to precede the reality and environment in which the organization may operate by creating a narrative that is based on current facts. As a result, scenario designing enables the identification of possible behaviours, risks, uncertainties, suggestions, stimuli, causes, effects and realities, in order to contribute to the orientation of the project activity and to provide creative insights that help in the construction of macro trends analysis [2].

Thus, the design culture of Strategic Design is based on the orientation of creative techniques that transcend just designing a product, that is, plans that contemplate the interaction between all the constituents of a company (designers, services, consumers, etc.) for proposing an innovative value-based product-service system.

Turning now to the understanding of UX Design, we can draw on Kujala et al. [7], which places UX Design as the area of study that highlights the user, the context and the system, working on the emotional side and all usability and interaction aspects of this relationship, since UX is an acronym derived from it. English User experience, i.e. user experience [8].

Its main objective is to communicate with purpose and deliver real and effective results by recognizing the main motivations, needs and purpose of its users, aligning them with the company's objectives. In the process of project execution by UX arrays, the designer collects a wealth of valuable information about consumers, their habits, and other elements that need to be better developed. The intent of this procedure is to reach a wider audience and meet the needs of not only current but also future consumers [9].

We can say that UX can be deepened in various fields of study, always seeking to communicate consistently, working the particularities of its audience and crossing their points of contact with the company. Garret defends such an argument when he treats UX as the way to experience a product or service in the real world, saying that this experience is imbued with meaning only when they interact with a person - or a group of them - in a way. match and link meanings to your needs.

Another important element to note is that UX seeks to identify touchpoints and narrow differences in order to make it easier to understand a system so that it is fluid, intuitive and as adaptable as possible among a group of users. To ensure that this happens, the designer needs to think about each point of contact, ensuring that every detail aligns the purpose and needs of the brand with what its consumers expect.

Roher C [10], points out that UX corresponds to a wide range of questions and that, to know which methods are best suited to the work, the author maps them in three dimensions, emphasizing that the key issue for choosing the methods is evaluated what to 
do and at what time. The first dimension is "Attitudinal versus Behavioral," which can be understood by choosing methods that distinguish between what people say and what they do, in order to understand and measure their tastes, beliefs, and preferences. The second dimension comprises qualitative and quantitative analysis. The qualitative approach uses methods that collect behavioral data based on direct contact with the user. In quantitative terms, this data is collected indirectly, through data analysis tools for example. The third and final dimension deals with the context of use, which is to analyze how and if the study participants/target audience is using the product.

We can also understand that by designing products and delivering user-centric services that extrapolate functionality or simply bring new excitement, UX Design will be fulfilling its role.

Finally, we resume the design culture through the speech of Borba, et al. [11] where the authors state that the same allied to design [12]. It is able to develop by continually studying what in the commodity constitutes stimulus and motivation, meaning and dynamics of enjoyment, to the point that it can continually generate updates on the identity of the commodities. That is, innovation, design, produces new values for the consumer and the company.

From this point, we can emphasize two important roles for design (whether strategic or UX), and its culture to be truly effective: the user and the designer. It is understood that the role of the designer is to collect and analyze the signals emitted by people, thus being the interpreter of signs of sociocultural transformations and, consequently, of motivations, desires and hidden needs of users. Finally, he will be able to visualize the problems and develop approaches that model his solution by creating interconnections and interrelationships between the generated hypotheses to propose the possibilities to be explored. Thus, it is possible to understand how the user appropriates artefacts, how he behaves, diffuses and expresses his identity and tastes. In this context, the UX tools support the understanding of the signals identified/analyzed and interpreted by the designer. Still, in this sense, it is important to seek to insert users in the creation process so that a product-service system logic that works and consolidates this relationship can be elaborated, bringing the design closer to people, putting them beyond the role of consumers. This broad practice highlights the participatory practice of the process of projecting and producing meanings.

\section{Metaproject And UX in the Development of the Fashion Product-Service System}

We will now seek to develop a systemic model based on a project culture from the perspective of Strategic Design (DE) and UX Design, aiming also to integrate sectors of a company and guide them towards the same goal. However, we emphasize that the methods adopted here aim to plan the company and its actions strategically even before its actual application, focusing the work on the management and communication of the brand to reach the goals of the company and its users in a way concise and aligned throughout the product-service system offered.
According to Reyes, et al. [13], we understand that a strategic design-driven product-service system implies building value and increasing competitiveness through innovation not only of the product but of all the processes and dimensions that involve construction of a value chain, because the product-service system “(...) adds (...), services and experience to the process of its use. The system is enhanced by the strategic vision that considers variables internal and external to the organization (...)".

Such dimensions, however, require the use of a set of research tools and methods. The design branches chosen for this study and, consequently, their design cultures, comprise several methods and tools that seek to analyze, enhance and relate the internal and external forces of a system to identify its problems and then design solutions. We also understand that the intersection of these methodologies requires the participation of both those who work in the company and its end users, thus following a system of horizontal integration, without defining a hierarchy, that is, where everyone's interaction is equally important.

Pastori DO, et. al [14] teach that all this is part of systemic thinking that is characterized as a procedural way of identifying, analyzing and shaping organizational problems. Representations of solutions to such problems can be identified by the relationships between the parts of a given system, highlighting the interactions of its components.

Another important point to be emphasized is the role that the designer plays, as it will be up to him to establish and manage the dialogue between the areas that make up the company so that he guides and interprets ideas and reflections through design, which ends up correspondingly. a disruption of fixed, permanent and splitsector theoretical management models determined to perform only their function [14].

Therefore, in order to constitute a proposal that articulates the principles and tools of DE and UX Design, we need to align the design steps. In order for these steps to be carried out effectively, it is important to understand how they fit and function within an organization so that we can configure a flexible, interdisciplinary and constantly adapting methodology. From this organization, the design both provides new perspectives of strategic development for the organization and can evolve to a plan where it is possible to constantly review aspects corresponding to the characteristics of the design. (...) Not only can but also be the competence of design the development of management processes that lead to a profound revision of the own figure of the designer [14].

It is also important to emphasize that the application and use of objective-oriented techniques, tools and methods correspond to a design methodology where creativity and practical applicability are not nullified, being characterized as a set of techniques and methods that seek to help the designer to enable solutions of a problem, being more articulated (not having a linearity), since each project is different and, therefore, the established projection order is molded from the problem identification (briefing). 
Thus, the relevance of this intersection of methodologies is presented from the restlessness and questions made by established methods, so that it allows to move in different areas of knowledge so that new perspectives of approach and exploration of design possibilities emerge. However, it is noteworthy that some questioning matrices are maintained, but the approach taken from them is much more fluid, allowing systemic thinking and learning from the rescue of action and reflection on the act of designing, rescuing their strategic and open innovation dimension [14].

Understanding the logic behind design-driven design methodology, we have defined key macro steps for organizing systemic thinking and for deepening and defining techniques and strategies that can be adopted at each stage of designing a fashion product-service system. Thus, as a starting point, we have the definition of the macro steps from the DE and UX Design, which are characterized respectively as meta-projectual, design, application and monitoring.

We saw that the meta-projectual phase corresponds to a moment of ideation and problematization/problematization of the brief, being the briefing responsible for establishing some design parameters, starting the design task [15].

From the briefing begin to emerge the first qualitative and contextual research, which aims to explore ideas that serve as a source of creativity, identify how the problem is perceived in the market and what issues it also involves, beyond the marketing perspective. This is a qualitative step, and as such, it utilizes research tools that evaluate and qualify motivations and behaviours such as trend analysis, creative stimuli, blue sky, super constant, interviews, focus groups, case studies and so on. , open the problem to possibilities for innovation [4].

After completing the briefing and its research, we can use the Scenario development tool, according to DE. The creation of scenarios enables the anticipation and simulation of future realities, helping and considering market, society and culture variables, also serving as a way of thinking about preventive actions for future problems. This technique can be used to set parameters for a search or after searches. Concluding with Pastori, et. al [14] scenarios are an important step in the projection process, as they stimulate, through mental representations usually expressed by symbolic and imagery inputs, aspects that can affect organizations. That is, the scenarios also serve to think actions and processes, potentially reinventing, often, an organization's own business plan.

Then, we chose to build a Focus Group as a technique for qualitative data collection of users, so that it helps the metaprojectual phase and retakes the dimensions of UX signaled by Roher. The focus group is, as defined by Backes, et. al [1], a research source that allows the collection of information from a dialectical perspective, where the group has common goals and its participants try to approach them by working as a team. In this conception, there is an intention to sensitize the participants to operate in the transformation of reality in a critical and creative way.
To organize such directions and ideas that will depart from the notes made by users in a focus group, and conclude the metaprojectual phase, we also chose to use a conceptual map, a tool that structures the main key concepts, connecting and highlighting the relationships and developments. of the words in common [17].

Importantly, in some cases, after research with users, there may be a redirection of the original briefing, making a review to explore other possibilities of problem. From the perspective of Strategic Design, this practice called counter-briefing is a dynamic methodological device, as it allows for an open systemic character due to the inherent perspective of problem review / initial briefing. This practice establishes a dynamic meta-projectual process, where reflection movements can focus on the review, reevaluation, and agile repositioning of an organization's complex strategies [18].

We understand, therefore, that dismembering the problem, researching and analyzing the current and possible scenarios, generating visions and abstracting concepts, stimulates the creative development of references and gives the basis for the conception of products and services that are relevant to the company and to people. This design logic also resizes the designer's performance, shifting his practices from the operational scope of organizations and makes him an agent of strategies, which rethinks and resizes the problems of the organization.

Here, once again, it is possible to identify the strategic and open character of the systemic model that we are building, since it is possible to resume phases prior to the projection itself, assessing whether the data obtained are sufficient to continue the conceptualization and definition and organization of techniques that will help the choice of actions to be implemented in the project.

Thinking about the size of the product-service system of a userbased fashion brand and its experience, this system is basically fueled by the desires, needs, likes and rejections of its users. The designer will work to interpret the demands, uses and interactions between consumers and the system-product, seeking to encompass potential relationships with their preferences.

In order to make these principles tangible, our design proposal begins with brainstorming and visual brain dumping. Brainstorm is a free expression tool that allows the designer to define initial concepts to start the project, producing quick connections and questions, expanding his thinking and empowering unusual ideas through word choice [19]. Visual brain dumping can be understood as the visual way to illustrate a brainstorm. They are similar techniques, however, Brain dumping explores quick and intensive sketches about signs, images, symbols and shapes that can be important visual elements to explore in the project.

Next, as a way of organizing words and visual elements, we will make a mind map where we define a central concept that branches connecting and structuring ideas and elements that can be harnessed for communication, design, experience, services and products [19]. After completing the mind map, and with the most outlined design guidelines, one begins to outline strategies and 
define the fundamental characteristics of projects through the first sketches.

Because of UX Design and the goal of building the entire brand dimension based on elements that converge with users, we first defined our audience by building Personas. Persona building is a technique that creates representations of real users who, unlike audience delimitation (which defines broad and generalized data of people who may want your product), explores particularities defined from "behaviors, needs and motivations [...] based on insights drawn from research" [10] that makes designers create bonds, empathy and better understand consumers during the design process.

From the relationship between the mind map and the Persona, Forced Connections can be established, a tool that, in turn, corresponds to the act of combining unpredictable functions and services to generate innovation. One way to create these connections is by overlapping ideas that imply the design problem with the universe of things that are part of everyday life or user needs, creating mood boards helps to visualize the associations between elements through a composition with images, keywords and concepts, color palettes and textures. The mood boards synthesize the concepts and the atmosphere to be achieved, which helps the designer in the search for partnerships and in developing experiences and services that can be linked with the brand [20].

From the results obtained by these methods, it is already possible to conceptualize a brand identity, the audience that it will serve and its positioning, as well as glimpse the viability of the product-service system linked to the experiences.

To complete the design macro step and define the visual aspects of brand identity, a moment begins to generate alternatives created from Sketches. Sketches are the beginning of the Prototyping process. Through them, even if less detailed and simpler, the designer is able to shape options to reach the desired results allowing the notion of the elements that will be explored [21].
Starting from the sketches, begins the Execution step, where everything that was built begins to be tangible. At this stage, we will rely on prototypes and mock-ups, once we understand that these are the simulations that come closest to the end result.

Prototypes and mock-ups serve the purpose of verifying the aesthetic-formal properties of objects, their proportions and their details and evaluating, visualizing the product more effectively. They combine the perspective of Prototypes, as they are tangible experiments. However, mock-ups can be digitally built. Thus, one facilitates the visualization of the fashion product for example and another of the company's visual identity. At this stage, it is also possible to identify the limitations of feasibility and then revisit the initial concepts to identify other paths can be explored to fine-tune the results, given the aspects that are considered vital in the project.

Therefore, if Prototypes are approved, they become final results and, once they are placed on the market or tested with users even before their actual application, need to be monitored to analyze their functioning, if the objectives Outlines in the meta-project phase are being met, if user perceptions match and planned strategies are being realized. There are ways to qualitatively verify these aspects, especially regarding the individual experience of each user. UX Design's own Consumer Journey Map provides an overview of all touchpoints of user engagement with the brand from its interaction with the entire dimension of the product-service system. This allows the designer to map out what motivated the consumer and how he or she reached the brand and does so or prevents them from re-relating to it. From this observation arise identifications of new problems for projection and opportunities for innovation [10]. This system is fed by user feedback that can be obtained through customer service channels and the user's own direct relationship with the company, such as email and social networks, for example.

All the methodological logic we have built so far has been synthesized into a conceptual model, comprising macro steps and tools. This model can be seen in Figure 1.

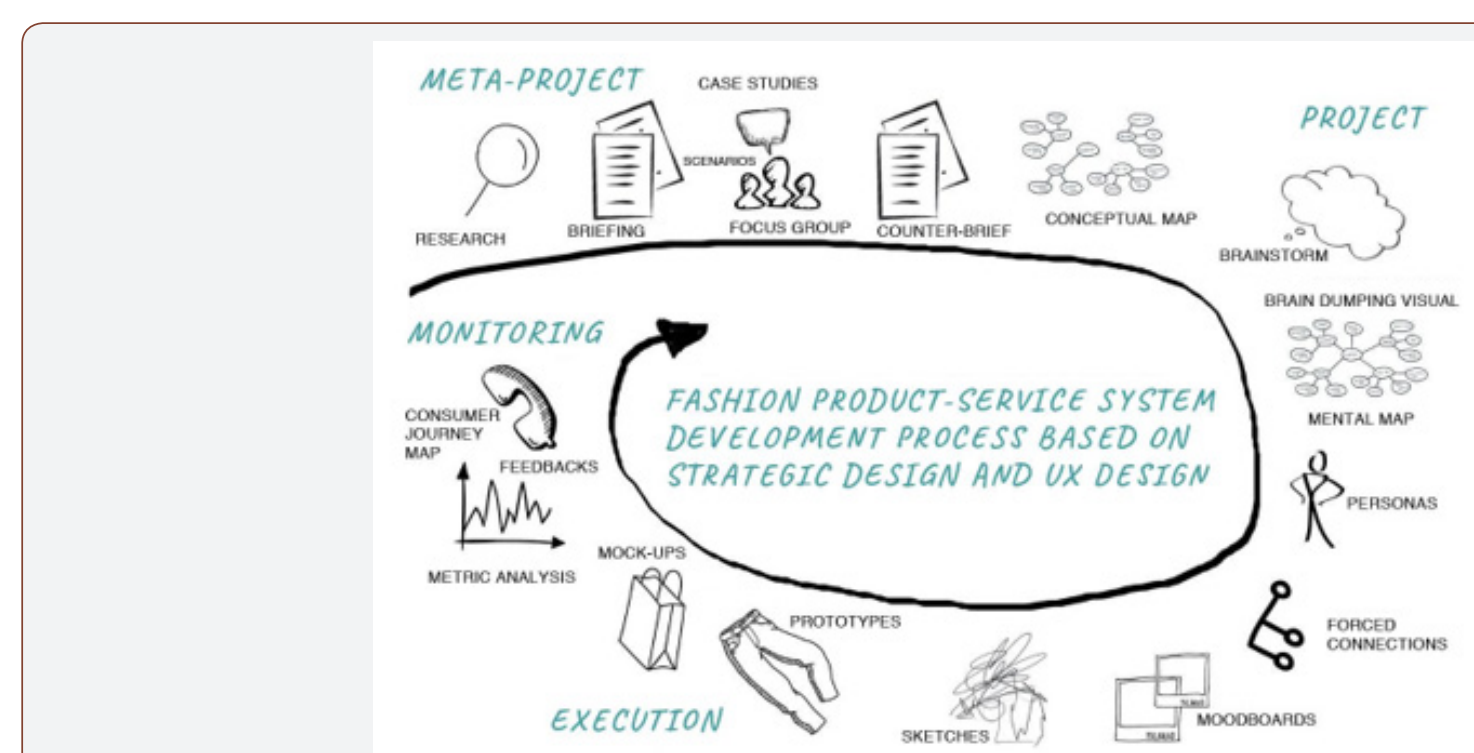

Figure 1: Systemic model of product-service system development of a fashion brand based on Strategic Design and UX Design. 
It is important to highlight that the purpose of this article is not to dictate that this methodology is the most correct and appropriate for thinking about a fashion project, nor does it guarantee that it will be a success or fail-proof, but rather to seek to analyze how systemic thinking Inspired by Strategic Design and UX Design, it provides insights, creative tools, and techniques that help you develop a fashion brand creatively and explore a design culture.

\section{Final Considerations}

Inspired by the thought of Pastori et. al [14] sought to contribute and stimulate methodological critical reflection, understanding that there are numerous tools, processes and design techniques and that the relationship between the market and man is often something complex to analyze, especially the from a linear design bias. In this sense, the study also aims to question the reductionist view that design is only an operational discipline, showing that it allows to expand and encompass its panorama of visions and disciplines, thinking and positioning strategically and producing meanings. Ultimately, this systemic thinking generated from the two selected design areas (DE and UX Design) will materialize in a logic that supports a design culture that can strategically fit into the field and fashion market, and not just thinking about goals and results.

For Celaschi, et al. [3] this reinforces the exercise of the strategic role of the designer, seen as a mediator between the interaction of the current market, which is demanding and sophisticated and saturated with products, taking into account the significant number of critical consumers. As this is a complex and indispensable task for the development of a contemporary brand, the designer ends up being a professional fully capable of performing a strategic practice in different organizations.

In conclusion, the potential relationships between DE and UX Design emerge from their natural essences of developing beyond design practice, addressing new competency dynamics absorbed from other areas to provide new perspectives on problem approaches and guide them. to the same goal. Moreover, they aim to consider the organization, management, experience, communication, analysis and synthesis to constitute a concise strategy and provide the feasibility of delivering meaningful and meaningful results, valuing the role of designers and also of people to which the proposals are being thought of [13].

\section{Acknowledgement}

None.

\section{Conflict of Interest}

Authors declare no conflict of interest.

\section{References}

1. Krippendorff K (1989) On the Essential Contexts of Artifacts or on the Proposition That “Design Is Making Sens (of Things)". Design Issue 5(2): 9-38.

2. Dijon de M (2010) Metaprojeto: o design does design. Blucher.

3. Franzato C (2010) Strategic design in the dialogue between design culture and company culture. Strategic Design Research Journal 3(3): 89-96.

4. Zurlo, Francis (2011) Strategic Design. In: $21^{\text {st }}$ Century, vol 6, Spaces and the Arts. Rome: Encyclopedia Treccani.

5. Henry M, Bruce A, Joseph L (2000) Strategy Safari. Porto Alegre: Bookmann.

6. Cardoso R (2007) The coded World: A philosophy of design and communication. Cosac Naify (edt).

7. Kujala S, Roto V, Vainio-Mattila KV, Karapanos E, Sinnelä A (2011) UX Curve: A method for evaluating long-term user experience. Interacting with Computers 23(5): 473-483.

8. Fabricio T (2014) Introduction and Best practices IN UX Design. Editora Casa do code.

9. Raffcom (2016) UX Design Practice areas. Brusque.

10. Rohrer C (2014) When to use which user-experience research methods. Nielsen Norman Group.

11. Henry M, Bruce A, Joseph L (2000) Strategy Safari. Porto Alegre: Bookmann.

12. Garrett J (2011) The Elements of User Experience: User-Centered Design for the Web and Beyond. Berkeley: New Riders.

13. Severo G, Reyes, Belo P (2007) Design-driven Innovation: The construction of an innovation-oriented organization. In: International Congress of Research in Design: 1-8.

14. Pastori DO, Merker F, Lopes JM, Sezera J, Montaña MP, et al. (2009) Strategic management process design and the systemic thought: The emergency of new design management methodologies. Strategic Design Research Journal 2(1): 11-23.

15. Phillips PL (2007) Briefing: a gestão do projeto de design. Blucher.

16. Backes, Stein D (2011) Focal Group as a technique for data collection and analysis in qualitative research. The world of Health, 35(4): 438-442.

17. Novak JD (2006) The theory underlying concept maps and how to construct them. Florida Institute for Human and Machine Cognition: Pensacola, USA.

18. Pastori DO, Merker F, Lopes JM, Sezera J, Montaña MP, et al. (2009) Strategic management process design and the systemic thought: The emergency of new design management methodologies. Strategic Design Research Journal 2(1): 11-23.

19. Ellen L (2013) Intuition, action, creation. São Paulo: Gustavo Gili.

20. Raffcom (2016) UX Design Practice areas. Brusque.

21. Botelho Tavares SR (2013) UX Entertainment content Management Platform: Design of the prototype research. 